\title{
THE THEORY OF COLLOID CHEMISTRY ${ }^{1}$
}

\section{BY WILDER D. BANCROFT}

We have dropped Graham's view that a colloidal substance must be amorphous, and we now call a phase colloidal when it is sufficiently subdivided, not committing ourselves definitely as to what degree of subdivision. Colloid chemistry is, therefore, the chemistry of bubbles, drops, grains, films, and filaments. Consequently the differences between colloid chemistry and the chemistry of relatively large masses must be differences arising from the subdivision of a phase or phases. I shall try to make clear that this line of attack leads us to a fairly consistent theory of colloids, a thing which we have not had before.

We begin, as Freundlich has done, with the properties of surfaces. A solid or a liquid surface has the power of adsorbing solids, liquids or gases. The familiar instance of this is the formation of an air film or water film on the surface of glass. We now postulate that surfaces show selective adsorption. This enables us to account qualitatively for all the phenomena of adsorption from solutions including the decomposition. If a substance adsorbs a given base much more readily than a given acid, there will be a tendency for the salt of that base and acid to hydrolyze and for the base to be taken up, leaving the solution acid. The quantitative side of the adsorption is given approximately in simple cases by some form of an exponential formula, the so-called adsorption isotherm. Since we have to have a qualitative theory before we can get a quantitative one, the chief use of the adsorption isotherm for the present is to enable us to tell that a definite compound is not formed. Adsorption is always reversible theoretically, though this is often apparently not true experimentally. If charcoal takes a dye out of solution

${ }^{1}$ A paper read at the symposium on colloid chemistry during the Montreal meeting of the American Chemical Society. 
to such an extent as to decolorize the water practically completely, no amount of washing with pure water will give a colored solution. ${ }^{1}$ In such a case the adsorption is strictly reversible and the difficulty was in the interpretation of the results. If the adsorbing substance changes either through agglomeration, crystallization, etc., its specific adsorbing power will change and the adsorbed substance will be held either more firmly or less firmly as the case may be. Either way we get an apparent irreversibility. Both cases are known. ${ }^{2}$ The adsorbed substance may also change on standing or on heating, in which case we shall have an apparent irreversibility though we are really dealing, as before, with a new, reversible equilibrium. This case occurs very frequently in dyeing.

It is perfectly familiar to all of us that small drops of a liquid will coalesce to a single large one when brought in contact. It is not familiar to us that solids also coalesce when brought in contact, because solids, as we know them, usually have a film of air on the surface and we do not bring them in contact at all. In fact Cushman and Coggeshall ${ }^{3}$ report that in their work a powder which would pass through a 200 mesh sieve surged like a liquid. The film of air was so large relatively to the mass of the particle that they were really dealing with little elastic balls having a hard core. If it were not for the air film, a broken plate could be mended at once by pressing the fragments together. ${ }^{4}$ Heating drives out the air film and also tends to make the solid more malleable, so that pressure brings more points in actual contact. We can join two pieces of glass while they are still very viscous and we join two pieces of platinum in the laboratory at temperatures far below the melting point of platinum. The import-

${ }^{1}$ Freundlich and Neumann: Zeit. phys. Chem., 67, 538 (1909).

${ }^{2}$ Cameron and Patten: Jour. Phys. Chem., II, 581 (1907); Lottermoser: Zeit. phys. Chem., 62, 377 (1908); Freundlich and Schucht: Ibid., 85, 660 (I9I3).

${ }^{3}$ Jour. Franklin Inst., 174, 672 (1912).

${ }^{4}$ Cf. Brewer: Kitte und Klebstoffe, 24 (1907). 
ance of clean surfaces when soldering is well known. We can electroplate copper on copper without difficulty because we can keep a clean surface. To plate an adherent deposit of nickel on nickel requires special precautions; and it is a much more difficult matter to get an adherent deposit of gold or silver upon aluminum.

We, therefore, conclude that solid grains or liquid drops will agglomerate if actually brought into contact and that to keep them from doing so we must have a surface film of some sort. Since this agglomeration takes place spontaneously, work must be done on the liquid or solid to disintegrate it. It is easy to see also that a film might keep drops separated and yet not break up a large drop into a lot of smaller ones. Dirty mercury will remain as a single liquid mass indefinitely so far as we know. If we disintegrate the mass by shaking, a film will form over each drop and will keep the drops from coalescing. It is also easy to see that less work will be necessary "to disintegrate the mercury in case we add something which lowers the surface tension of the mercury. In some cases we get disintegration very easily. Water will disintegrate gelatine at about $30^{\circ}$; but cellulose only at very much higher temperatures. The disintegrating action of water on metals is negligible except under special conditions. ${ }^{1}$ Except with such substances as gelatine we have to use special methods of disintegration, and the presence of a suitable surface film of some sort is necessary to prevent the liquid drops or solid particles from coalescing again.

If we drop a stone into water, it sinks very rapidly; if we grind the stone into coarse particles these sink less rapidly; if we grind the stone into fine particles, these sink slowly; if we grind the stone into very fine particles we should expect them to sink very slowly, the rate being a function of the diameter of the particles. This is not the case, however. Very fine particles do not follow Stokes' equation and do not (1912).

1 Mengarini and Scala: Zeit. Kolloidchemie, 6, 65, 240 (1910); 10, I I3 
settle at all, because of the Brownian movements which are negligible for coarse particles. We, therefore, conclude that any substance can be brought into a state of colloidal solution provided we make the particles of that phase so small that the Brownian movements will keep the particles suspended, and provided we prevent agglomeration of the particles by a suitable surface film. Coalescence may be prevented by a non-electrical film, by an electrical film (electrical charge) or by any combination of the two. Non-electrical suspensions are due to marked adsorption of substances in true solution together with a suitable change of surface tension-to be discussed under peptonization-or to the adsorption of a colloid which is readily peptonized by water, a protecting colloid so-called. It is not proper to assume, as Freundlich ${ }^{1}$ does, that all adsorption is accompanied by a lowering of the surface tension. Freundlich bases his conclusions on the Gibbs' formula which applies to a homogeneous solution and not to the case of adsorption at all. An electrical suspension is due to the preferential adsorption of some ion. So long as the particles are all charged positively or all negatively they will repel each other and will not coalesce. Neutralization of the charge causes precipitation through agglomeration.

On this statement of facts there is no apparent reason why one should not make a colloidal solution of any substance of almost any desired concentration. Practically, we cannot do this, though we can vary the concentration within limits by changing the protecting colloid. Also, we know that people determined the solubility of tannin in water when they thought that it really dissolved. We know now that tannin only appears to dissolve in water; but a change in our theoretical views cannot change the facts, though it may change our interpretation of them. I, therefore, conclude that there is a limiting concentration beyond which the peptonizing action of the liquid and the protective action of the surface film cease to be effective and that we consequently get an apparently

${ }^{1}$ Kapillarchemie, 52 (1909). 
definite solubility which, of course, varies with the size of the particles. In fact, I suspect that a number of solubility determinations on such things as the so-called calcium sucrates will turn out to have been really a study of colloidal solutions. In view of what we know in regard to the behavior of the cellulose nitrates with mixed solvents, I doubt whether Oudemanns ${ }^{1}$ determined the true solubility of cinchonine in alcoholic chloroform.

If we start with two liquids, either can be dispersed in the other and the question of which constitutes the internal phase depends entirely on the nature of the protecting film. For an emulsion to be possible the third substance must pass into the surface separating the liquids ${ }^{2}$ and must possess the power of forming a coherent film in presence of the two liquids. Let $A$ be one liquid, $B$ the other, and $C$ the protecting film. If the surface tension of $C$ is lower on the side towards $B$, liquid $B$ will tend to form the internal phase, while liquid $A$ will tend to form the internal phase if the reverse be true. ${ }^{3}$ This has been realized experimentally in a number of cases. ${ }^{4}$ With a sodium soap, oil becomes the internal phase and water the external one, while the reverse is true with a calcium or magnesium soap. Rosin enables one to emulsify large amounts of water in linseed oil.

The study of emulsions throws light on the more difficult question of peptonization. Doyle ${ }^{5}$ found that iodine was adsorbed readily by charcoal or graphite and yet had very little effect in preventing sedimentation, while other substances were adsorbed strongly and had a marked effect in preventing sedimentation. Consequently adsorption of two different substances may act in fundamentally different ways. This is true irrespective of whether any or all of the three substances are or are not electrolytes. As yet there are no experimental

\footnotetext{
1 Zeit. anal. Chem., II, 287 (1872).

${ }^{2}$ Hofmann: Zeit. phys. Chem, 83, 385 (1913).

3 Bancroft: Jour. Phys. Chem., I7, 5r5 (1913).

4 Newman: Ibid., 18, 34 (1914).

5 Jour. Phys. Chem., I7, 390 (1913).
} 
data on this point; but it seems probable that a relatively low surface tension on the water side of the adsorbed film and a relatively high surface tension on the side towards the solid will make the film curl so as to tend to peptonize the solid as internal phase. If the reverse is the case the solid will tend to become the external phase. Neither tendency will prevail if the two surface tensions are practically equal. I hope to test this before long with pyroxylin and mixed solvents.

All methods for preparing colloidal solutions can be classified either as condensation or as dispersion methods. Condensation methods involve precipitation as finely divided particles and suitable strong adsorption (including protecting colloids) or precipitating agents kept low either by suitable choice of reagents or by dilution. Dispersion methods involve the washing out of precipitating agents (effective only so long as particles are finely divided), the addition of a peptonizing agent (suitable strong adsorption), or disintegration by electrical, mechanical, or other means in the absence of a precipitating agent or in the presence of a protecting agent.

The properties of colloids with water as external phase follow readily from the theory outlined. If the suspended particles are practically insoluble, there will be no change of surface tension, no osmotic pressure and no diffusion except what is due to the Brownian movements. If the particles are slightly soluble, there will be a slight change of surface tension, some osmotic pressure, and some diffusion other than that due to the Brownian movements. In so far as the colloids are electrical suspensions, they will move to the cathode or anode, depending on whether they adsorb a cation or anion preferentially. Since we are dealing throughout with selective adsorption, we see that at best it can only be a first approximation to group all univalent cations together and all bivalent cations, etc. As a matter of fact, the data cited by Freundlich ${ }^{1}$ to show the desirability of such a grouping constitute a still ' stronger argument against it. The data of

\footnotetext{
${ }^{1}$ Kapillarchemie, 351, 352, 367 (r909).
} 
Pappadà show that one must discriminate between the different alkali metals. We have always put hydrogen and hydroxyl ions in a separate class because it was quite impossible to group them with the other univalent ions. Potassium and nitrate ions do not peptonize silver bromide perceptibly; but silver and bromine ions do. The moment we consider the matter as one of selective adsorption, we see that one cannot ignore the nature of the anions in solution when we are dealing with a negatively charged particle. We have been misled by an injudicious selection of salts. As I pointed out at the Cincinnati meeting, the theory of selective adsorption enables is to predict the reversal of the effects of the anions on the stability of albumin when one changes from a slightly alkaline to a slightly acid solution.

Positive and negative colloids will, of course, precipitate each other when in proper relative proportions, and provided adsorption takes place. These precipitates may be peptonized as such under suitable conditions and may then easily be mistaken for compounds. The lakes are one instance of this and I imagine that aluminum silicate is another, when precipitated in the wet way.

Since any substance may be converted into a colloidal suspension by being subdivided sufficiently, ${ }^{2}$ it follows that we may have any number of different colloidal solutions of the same thing if we start, for instance, with ferric oxide or aluminum oxide having different amounts of water in the particles. As a matter of fact we have recognized for years at least two different colloidal iron oxides and colloidal aluminas. Nobody knows what we have in the case of the cellulose nitrates. If a precipitated suspension agglomerates on standing, it will not peptonize with the same readiness as before or with the same concentration of peptonizing agent. If the agglomeration is accompanied, as it often is, by a change in water content, we have two variables each of which may have a marked

\footnotetext{
${ }^{1}$ Zeit. Kolloidchemie, 4, 214 (1909); 6, 83 (I910); 9, 136 (I9I1).

${ }^{2} \mathrm{Cr}$. Wegelin: Zeit. Kolloidchemie, 14, 65 (1914).
} 
effect on the peptonization or apparent solubility. Many instances of this are known especially with sulphides and hydroxides so-called (including silicic acid).

No distinction has been made thus far between colloidal suspensions and colloidal solutions ${ }^{1}$ because I believe that this distinction is an unsound one though I recognized the usefulness of it at the time it was made. While we are reasonably sure that colloidal gold is a solid and that colloidal oil is a liquid, the two behave exactly alike when both are suspended electrically. ${ }^{2}$ There is no apparent reason why we should put hydrous ferric oxide on one side of the line and hydrous stannic oxide on the other. I fail to see any fundamental difference between a chromic oxide jelly and a silicic oxide jelly. Consequently, I believe that we should drop the terms of suspension colloids and emulsion colloids, which are now a hindrance and not a help. On the other hand, there is a difference between a colloidal solution in which the more mobile phase (usually water) is external phase and one in which the water is internal phase.

I have already pointed out the properties of colloidal solutions with water as external phase and I will now take up the case where the more mobile phase is internal. We must have a honeycomb structure and we may, therefore, get a distinct change in surface tension and in viscosity, In so far as the less mobile phase is practically insoluble in the other we shall get no appreciable osmotic pressure. We get these phenomena to a marked degree with gelatine and I conclude that gelatine is usually the external phase in a so-called aqueous solution of gelatine. ${ }^{3}$ These two types, which correspond to a certain extent to the suspension colloids and emulsion colloids, are not to be considered as hard and fast things. We can change from an emulsion of oil in water to one of water in oil by changing the nature of the third component. Under

${ }^{1}$ Noyes: Jour. Am. Chem. Soc., 27, 85 (1905).

${ }^{2}$ Ellis: Zeit. phys. Chem., 80, 597 (I912).

3 This was first suggested to me by Mr. T. R. Briggs three or four years ago. 
peptonization we saw that one adsorbed substance tends to peptonize the solid as external phase and another as internal phase. It might be very difficult to find a substance that would peptonize gold as external phase in water; but there should be no difficulty in changing from one type to the other if we took less extreme cases. Concentrated sodium sulphate. solution flocculates gelatine instead of making it set to a jelly and we can get hydrous ferric oxide coming down either as unquestionably internal phase or as a jelly in which it probably is external phase. Since some of the colloids which form the external phase are readily dispersed by water (gelatine for instance), the solutions are fairly stable. All gradations are known, however.

Jellies may have a honeycomb structure, in which case the water is internal phase and the gelatinous matter constitutes the external one; or it may have a sponge structure. It is usually assumed that in this latter case the water is the external phase, apparently because a sponge can be immersed completely in water. A moment's thought will show, however, that there is neither external nor internal phase. Each phase is continuous and neither is dispersed in the other. This will be seen a little more clearly perhaps if we consider a roll of wire fence standing out-doors. The fence is continuous and, therefore, is not the dispersed phase; but exactly the same is true about the air. The general opinion seems to be that most jellies are of the sponge type and are formed by the coagulation and partial coalescence of a dispersed phase. ${ }^{1}$ I doubt the accuracy of the conclusion. In the benzene-water and oilwater jellies, we know that we have a honeycomb structure. Wet sand would be a sponge structure if the grains of sand could be made to unite at the edges. Actually it is a case of water as the external phase. A brick is a solid-and-vapor jelly of the sponge type.

It is quite evident that a solution having a honeycomb

${ }^{1}$ Zsigmondy: Zeit. anorg. Chem., 7r, 356 (I9II); Bachmann: Ibid., 73, 125 (1912); Flade: Zeit. phys. Chem., 82, $\times 73$ (1913). 
structure can change readily into a jelly; but I do not see how a solution with gelatinous particles suspended in it can become a jelly without leaving a liquid layer. It seems to me possible to have a liquid layer formed when we have a honeycomb jelly; but that we must have a honeycomb jelly whenever there is no liquid layer formed. If a honeycomb jelly retains its flexibility of walls while drying, it will dry to a varnish. If the partially dried jelly takes up water again, it will swell. I can see how a honeycomb jelly can swell in water without being disintegrated, because the collapsed cells fill with water; but I do not believe that a sponge jelly can do this to any appreciable extent.

In the foregoing outline of a working theory of colloid chemistry only four postulates have been made, and the first three of these will be accepted by everybody without any hesitation.

I. Colloid chemistry differs from ordinary chemistry only through the variations resulting from the increasing dispersity of one or more phases.

2. Selective adsorption takes place at surfaces.

3. Sufficiently finely divided particles, which are prevented from coalescing, will be kept suspended in a medium by the Brownian movements.

4. Under fixed conditions of peptonization we get an approximately definite limiting concentration corresponding to a saturated solution in the case of a true solution.

Cornell University

1 Bancroft: Jour. Phys. Chem., 16, 395 (rg12). 